\title{
A STRUCTURE THEOREM FOR COMPLETELY REGULAR SEMIGROUPS
}

\author{
MARIO PETRICH
}

\begin{abstract}
Structure theorems for arbitrary completely regular semigroups have been given by Yamada, Warne, Petrich, and Clifford. A new structure theorem for these semigroups is proved here. It is reminiscent of both the structure theorem of Clifford-Petrich and of the Rees construction for completely simple semigroups. It simplicity ought to prove useful in the study of various aspects of completely regular semigroups.
\end{abstract}

Completely regular semigroups, also called unions of groups, along with inverse semigroups, represents one of the most promising classes of semigroups from the point of view of construction, properties, congruences, varieties, amalgamation,etc. Their global structure in terms of semilattices of completely simple semigroups was discovered by Clifford in an early paper [1]. Their local structure is described by means of the Rees theorem for completely simple semigroups [5] which also stems from the same time period. This took care of the coarse structure of completely regular semigroups.

A description of the interaction of the various completely simple components of a completely regular semigroup, that is its fine structure, had to wait quite a long time. After the structure of completely regular semigroups belonging to some restricted classes had been successfully treated, Yamada [7], Warne [6], Petrich [4], and Clifford-Petrich [2] came up with different constructions of a general completely regular semigroup. Unfortunately, all of these constructions are notationally quite complex.

We offer here a simple construction of completely regular semigroups. Its rudiments can be traced to [2] and incorporates an idea of J. A. Gerhard. Its setting is reminiscent of the Rees construction for completely simple semigroups with ingredients which are both few in number and transparent in nature. The surprisingly simple form of this construction should prove useful in diverse studies of completely regular semigroups, such as the structure of special classes, free objects, etc.

All necessary background, and in particular the notation and terminology, can be found in Petrich [3].

THEOREM. Let $Y$ be a semilattice. For every $\alpha \in Y$, let $S_{\alpha}=\mathcal{M}\left(I_{\alpha}, G_{\alpha}, \Lambda_{\alpha} ; P_{\alpha}\right)$ be a Rees matrix semigroup such that $P_{\alpha}$ is normalized at an element also denoted

Received by the editors November 25, 1985.

1980 Mathematics Subject Classification (1985 Revision). Primary 20M10.

Key words and phrases. Structure, completely regular semigroup, semilattice of completely simple semigroups, the Rees theorem. 
by $\alpha$ and suppose that $S_{\alpha} \cap S_{\beta}=\varnothing$ if $\alpha \neq \beta$. Let

$$
\begin{gathered}
\langle, \quad\rangle: S_{\alpha} \times I_{\beta} \rightarrow I_{\beta}, \\
S_{\alpha} \rightarrow G_{\beta} \text { in notation } a \rightarrow a_{\beta}, \\
{[, \quad]: \Lambda_{\beta} \times S_{\alpha} \rightarrow \Lambda_{\beta}}
\end{gathered}
$$

be functions defined whenever $\alpha \geq \beta$ and satisfying the following conditions. Let $a \in S_{\alpha}$ and $b \in S_{\beta}$.

(i) If $\alpha \geq \beta, i \in I_{\beta}, \lambda \in \Lambda_{\beta}$, then

$$
p_{\lambda\langle a, i\rangle} a_{\beta} p_{[\beta, a] i}=p_{\lambda\langle a, \beta\rangle} a_{\beta} p_{[\lambda, a] i} .
$$

(ii) If $i \in I_{\alpha}$ and $\lambda \in \Lambda_{\alpha}$, then

$$
a=\left(\langle a, i\rangle, a_{\alpha},[\lambda, a]\right) .
$$

On $S=\bigcup_{\alpha \in Y} S_{\alpha}$ define a multiplication by

$$
a \circ b=\left(\langle a,\langle b, \alpha \beta\rangle\rangle, a_{\alpha \beta} p_{[\alpha \beta, a]\langle b, \alpha \beta\rangle} b_{\alpha \beta},[[\alpha \beta, a], b]\right) .
$$

(iii) If $\gamma \leq \alpha \beta, i \in I_{\gamma}, \lambda \in \Lambda_{\gamma}$, then

$$
\left(\langle a,\langle b, i\rangle\rangle, a_{\gamma} p_{[\gamma, a]\langle b, \gamma\rangle} b_{\gamma},[[\lambda, a], b]\right)=\left(\langle a \circ b, i\rangle,(a \circ b)_{\gamma},[\lambda, a \circ b]\right) .
$$

Then $S$ is a completely regular semigroup such that $S / D \cong Y$ and whose multiplication restricted to each $S_{\alpha}$ coincides with the given multiplication. Conversely, every completely regular semigroup is isomorphic to one so constructed.

PrOOF. Let $S$ be as constructed above. We verify first associativity of multiplication. Hence let $\alpha, \beta, \gamma \in Y, \delta=\alpha \beta \gamma, a \in S_{\alpha}, b \in S_{\beta}, c \in S_{\gamma}$. Then

$$
\begin{aligned}
& a \circ(b \circ c)=\left(\langle a,\langle b \circ c, \delta\rangle\rangle, a_{\delta} p_{[\delta, a]\langle b \circ c, \delta\rangle}(b \circ c)_{\delta},[[\delta, a], b \circ c]\right), \\
& (a \circ b) \circ c=\left(\langle a \circ b,\langle c, \delta\rangle\rangle,(a \circ b)_{\delta} p_{[\delta, a \circ b]\langle c, \delta\rangle} c_{\delta},[[\delta, a \circ b], c]\right) .
\end{aligned}
$$

Applying condition (iii) to this situation, we obtain

$$
\begin{gathered}
a \circ(b \circ c)=\left(\langle a,\langle b,\langle c, \delta,\rangle\rangle\rangle, a_{\delta} p_{[\delta, a]\langle b,\langle c, \delta\rangle\rangle} b_{\delta} p_{[\delta, b]\langle c, \delta\rangle} c_{\delta},[[[\delta, a], b], c]\right), \\
(a \circ b) \circ c=\left(\langle a,\langle b,\langle c, \delta\rangle\rangle\rangle, a_{\delta} p_{[\delta, a]\langle b, \delta\rangle} b_{\delta} p_{[[\delta, a], b]\langle c, \delta\rangle} c_{\delta},[[[\delta, a], b], c]\right) .
\end{gathered}
$$

With the substitution $\alpha \rightarrow \beta, \beta \rightarrow \delta, \lambda \rightarrow[\delta, a], a \rightarrow b, i \rightarrow\langle c, \delta\rangle$ in condition (i), we obtain that the middle entries on the right-hand sides of (5) and (6) are equal. Therefore $a \circ(b \circ c)=(a \circ b) \circ c$ which verifies associativity.

We now fix $\alpha \in Y$ and prove that multiplication $\circ$ coincides with the given multiplication in $S_{\alpha}$. Indeed, let $a=(i, g, \lambda), b=(j, h, \mu)$ with $a, b \in S_{\alpha}$. Using condition (ii), we get

$$
\begin{aligned}
a \circ b & =\left(\langle a,\langle b, \alpha\rangle\rangle, a_{\alpha} p_{[\alpha, a]\langle b, \alpha\rangle} b_{\alpha},[[\alpha, a], b]\right) \\
& =\left(i, g p_{\lambda j} h, \mu\right)=(i, g, \lambda)(j, h, \mu)=a b,
\end{aligned}
$$

be asserted.

The multiplication in $S$ clearly indicates that $S$ is a semilattice $Y$ of semigroups $S_{\alpha}$. Each $S_{\alpha}$ is completely simple by the Rees theorem and hence $S$ is completely regular. In addition, $S / D \cong Y$. 
Conversely, let $S$ be a completely regular semigroup. Then $S$ is a semilattice $Y$ of completely simple semigroups $S_{\alpha}$. In view of the Rees theorem, we may take $S_{\alpha}=\mathcal{M}\left(I_{\alpha}, G_{\alpha}, \Lambda_{\alpha} ; P_{\alpha}\right)$ with $P_{\alpha}$ normalized at an element denoted by $\alpha$.

For $\alpha \geq \beta, a \in S_{\alpha}, i \in I_{\beta}, \lambda \in \Lambda_{\beta}$, we define the required mappings implicitly by

$$
\begin{aligned}
a(i, 1, \beta) & =(\langle a, i\rangle,,), \\
a(\beta, 1, \beta) & =\left(, a_{\beta},\right), \\
(\beta, 1, \lambda) a & =(,,[\lambda, a]) .
\end{aligned}
$$

We thus obtain the mappings as indicated in (1), (2), and (3), respectively. It is easy to see that the last entry in (7) and the first entry in (9) are both equal to $\beta$. Because of this and (7) we obtain from (8)

$$
a(\beta, 1, \beta)=\left(\langle a, \beta\rangle, a_{\beta}, \beta\right) .
$$

Further, $(\beta, 1, \beta) a=(\beta, x,[\beta, a])$ for some $x \in G_{\beta}$. Hence

$$
\begin{gathered}
(\beta, 1, \beta)[a(\beta, 1, \beta)]=(\beta, 1, \beta)\left(\langle a, \beta\rangle, a_{\beta}, \beta\right)=\left(\beta, a_{\beta}, \beta\right), \\
{[(\beta, 1, \beta) a](\beta, 1, \beta)=(\beta, x,[\beta, a])(\beta, 1, \beta)=(\beta, x, \beta)}
\end{gathered}
$$

and thus $x=a_{\beta}$. We have proved

$$
(\beta, 1, \beta) a=\left(\beta, a_{\beta},[\beta, a]\right) .
$$

In order to verify condition (i), we continue with the above notation. Then $(\beta, 1, \lambda) a=(\beta, x,[\lambda, a])$ and $a(i, 1, \beta)=(\langle a, i\rangle, y, \beta)$ for some $x, y \in G_{\beta}$. We now calculate

$$
\begin{aligned}
\left(\beta, p_{\lambda\langle a, \beta\rangle} a_{\beta} p_{[\lambda, a] i}, \beta\right) \\
\quad=(\beta, 1, \lambda)\left(\langle a, \beta\rangle, a_{\beta},[\lambda, a]\right)(i, 1, \beta) \\
\quad=(\beta, 1, \lambda)\left(\langle a, \beta\rangle, a_{\beta}, \beta\right)\left(\beta, x^{-1}, \beta\right)(\beta, x,[\lambda, a])(i, 1, \beta) \\
\quad=(\beta, 1, \lambda)[a(\beta, 1, \beta)]\left(\beta, x^{-1}, \beta\right)[(\beta, 1, \lambda) a](i, 1, \beta) \quad \text { by }(10) \\
\quad=[(\beta, 1, \lambda) a](\beta, 1, \beta)\left(\beta, x^{-1}, \beta\right)(\beta, 1, \lambda)[a(i, 1, \beta)] \\
\quad=\left[(\beta, x,[\lambda, a])\left(\beta, x^{-1}, \lambda\right)\right](\langle a, i\rangle, y, \beta) \\
\quad=(\beta, 1, \lambda)(\langle a, i\rangle, y, \beta) \\
\quad=(\beta, 1, \lambda)(\langle a, i\rangle, 1, \beta)[a(i, 1, \beta)] \\
\quad=(\beta, 1, \lambda)(\langle a, i\rangle, 1, \beta)[(\beta, 1, \beta) a](i, 1, \beta) \\
\quad=(\beta, 1, \lambda)(\langle a, i\rangle, 1, \beta)\left(\beta, a_{\beta},[\beta, a]\right)(i, 1, \beta) \quad \text { by }(11) \\
\quad=\left(\beta, p_{\lambda\langle a, i\rangle} a_{\beta} p_{[\beta, a] i}, \beta\right)
\end{aligned}
$$

which verifies condition (i).

In order to prove condition (ii), let $a=(j, g, \mu) \in S_{\alpha}, i \in I_{\alpha}, \lambda \in \Lambda_{\alpha}$. Then

$$
\begin{aligned}
& a(i, 1, \alpha)=(j, g, \mu)(i, 1, \alpha)=(j,,), \\
& a(\alpha, 1, \alpha)=(j, g, \mu)(\alpha, 1, \alpha)=(, g,), \\
& (\alpha, 1, \lambda) a=(\alpha, 1, \lambda)(j, g, \mu)=(,, \mu)
\end{aligned}
$$

which implies that $\langle a, i\rangle=j, a_{\alpha}=g,[\lambda, a]=\mu$ so that $a=\left(\langle a, i\rangle, a_{\alpha},[\lambda, a]\right)$, as required. 
For the multiplication formula (4) and condition (iii), it will be convenient to first consider the following case. Let $a \in S_{\alpha}, b \in S_{\beta}$, and $\gamma \leq \alpha \beta$. On the one hand, we have

$$
\begin{aligned}
a b(\gamma, 1, \gamma) & =a\left(\langle b, \gamma\rangle, b_{\gamma}, \gamma\right) \quad \text { by }(10) \\
& =a(\langle b, \gamma\rangle, 1, \gamma)\left(\gamma, b_{\gamma}, \gamma\right) \\
& =(\langle a,\langle b, \gamma\rangle\rangle, x, \gamma)\left(\gamma, b_{\gamma}, \gamma\right) \quad \text { for some } x \in G_{\gamma} \\
& =(\langle a,\langle b, \gamma\rangle\rangle, 1, \gamma)(\langle a,\langle b, \gamma\rangle\rangle, x, \gamma)\left(\gamma, b_{\gamma}, \gamma\right) \\
& =(\langle a,\langle b, \gamma\rangle\rangle, 1, \gamma)[a(\langle b, \gamma\rangle, 1, \gamma)]\left(\gamma, b_{\gamma}, \gamma\right) \\
& =(\langle a,\langle b, \gamma\rangle\rangle, 1, \gamma)[(\gamma, 1, \gamma) a]\left(\langle b, \gamma\rangle, b_{\gamma}, \gamma\right) \\
& =(\langle a,\langle b, \gamma\rangle\rangle, 1, \gamma)\left(\gamma, a_{\gamma},[\gamma, a]\right)\left(\langle b, \gamma\rangle, b_{\gamma}, \gamma\right) \quad \text { by }(11) \\
& =\left(\langle a,\langle b, \gamma\rangle\rangle, a_{\gamma} p_{[\gamma, a]\langle b, \gamma\rangle} b_{\gamma}, \gamma\right) \\
& =\left(\langle a,\langle b, \gamma\rangle\rangle, a_{\gamma} p_{[\gamma, a]\langle b, \gamma\rangle} b_{\gamma},[[\gamma, a], b]\right)(\gamma, 1, \gamma) .
\end{aligned}
$$

A symmetric argument will show that

$$
\begin{aligned}
(\gamma, 1, \gamma) a b & =\left(\gamma, a_{\gamma} p_{[\gamma, a]\langle b, \gamma\rangle} b_{\gamma},[[\gamma, a] \mid, b]\right) \\
& =(\gamma, 1, \gamma)\left(\langle a,\langle b, \gamma\rangle\rangle, a_{\gamma} p_{[\gamma, a]\langle b, \gamma\rangle} b_{\gamma},[[\gamma, a], b]\right)
\end{aligned}
$$

For $\gamma=\alpha \beta$, we have that

$$
\left(\langle a,\langle b, \alpha \beta\rangle\rangle, a_{\alpha \beta} p_{[\alpha \beta, a]\langle b, \alpha \beta\rangle} b_{\alpha \beta},[[\alpha \beta, a], b]\right) \in S_{\alpha \beta}
$$

which together with $a b,(\alpha \beta, 1, \alpha \beta) \in S_{\alpha \beta}$, (12), and (13) with $\gamma=\alpha \beta$, by weak cancellation in $S_{\alpha \beta}$ yields the multiplication formula (4).

Again let $\gamma \leq \alpha \beta$. For $i \in I_{\gamma}$, we obtain on the one hand,

$$
\begin{aligned}
a b(i, 1, \gamma) & =a(\langle b, i\rangle, x, \gamma) \quad \text { for some } x \in G_{\gamma} \\
& =[a(\langle b, i\rangle, 1, \gamma)](\gamma, x, \gamma) \\
& =(\langle a,\langle b, i\rangle\rangle, y, \gamma)(\gamma, x, \gamma) \quad \text { for some } y \in G_{\gamma} \\
& =(\langle a,\langle b, i\rangle\rangle, y x, \gamma),
\end{aligned}
$$

and on the other hand,

$$
a b(i, 1, \gamma)=(\langle a b, i\rangle, z, \gamma) \text { for some } z \in G_{\gamma}
$$

and thus

$$
\langle a,\langle b, i\rangle\rangle=\langle a b, i\rangle .
$$

A symmetric proof will show that for $\lambda \in \Lambda_{\gamma}$, we have

$$
[[\lambda, a], b]=[\lambda, a b] \text {. }
$$

Furthermore, it was established in (12) that

$$
a b(\gamma, 1, \gamma)=\left(\langle a,\langle b, \gamma\rangle\rangle, a_{\gamma} p_{[\gamma, a]\langle b, \gamma\rangle} b_{\gamma}, \gamma\right)
$$

which together with

$$
a b(\gamma, 1, \gamma)=\left(\langle a b, \gamma\rangle,(a b)_{\gamma}, \gamma\right)
$$

yields

$$
a_{\gamma} p_{[\gamma, a]\langle b, \gamma\rangle} b_{\gamma}=(a b)_{\gamma} .
$$


Relations (14), (15), and (16) show that condition (iii) holds as well.

As an illustration of how the above construction simplifies in the case of completely regular semigroups belonging to some special class, we give below a construction of orthogroups, that is orthodox (idempotents form a subsemigroup) completely regular semigroups. We include a complete proof as it seems sufficiently instructive.

COROLlaRY. Let $Y$ be a semilattice. For every $\alpha \in Y$, let $S_{\alpha}=I_{\alpha} \times G_{\alpha} \times \Lambda_{\alpha}$, where $I_{\alpha}$ is a left zero semigroup, $G_{\alpha}$ is a group, and $\Lambda_{\alpha}$ is a right zero semigroup, and assume that $S_{\alpha} \cap S_{\beta}=\varnothing$ if $\alpha \neq \beta$. For each $\alpha \in Y$, fix an element in $I_{\alpha} \cap \Lambda_{\alpha}$ and denote it by $\alpha$. Let

$$
\langle,\rangle: S_{\alpha} \times I_{\beta} \rightarrow I_{\beta}, \quad[,]: \Lambda_{\beta} \times S_{\alpha} \rightarrow \Lambda_{\beta}
$$

be two functions defined whenever $\alpha \geq \beta$. Let $G$ be a semilattice $Y$ of groups $G_{\alpha}$ in which multiplication is denoted by juxtaposition. Assume that for all $a=(i, g, \lambda) \in$ $S_{\alpha}$ and $b=(j, h, \mu) \in S_{\beta}$, the following conditions hold.

(A) If $k \in I_{\alpha}$ and $\nu \in \Lambda_{\alpha}$, then $\langle a, k\rangle=i$ and $[\nu, a]=\lambda$.

On $S=\bigcup_{\alpha \in Y} S_{\alpha}$ define a multiplication by

$$
a \circ b=(\langle a,\langle b, \alpha \beta\rangle\rangle, g h,[[\alpha \beta, a], b]) .
$$

(B) If $\gamma \leq \alpha \beta, k \in I_{\gamma}, \nu \in \Lambda_{\gamma}$, then

$$
\langle a,\langle b, k\rangle\rangle=\langle a \circ b, k\rangle, \quad[[\nu, a], b]=[\nu, a \circ b] .
$$

Then $S$ is an orthogroup such that $S / D \cong Y$ and whose multiplication restricted to each $S_{\alpha}$ coincides with the given multiplication. Conversely, every orthogroup is isomorphic to one so constructed.

ProOF. For the direct part, we let $S$ be as constructed above and will show that $S$ is completely regular and orthodox. Since each $S_{\alpha}$ is a rectangular group, that is, an orthodox completely simple semigroup, it suffices to provide the function $S_{\alpha} \rightarrow G_{\beta}$ defined whenever $\alpha \geq \beta$ and to prove that the conditions in the theorem are met and the two multiplication formulae agree.

For any $\alpha, \beta \in Y$ such that $\alpha \geq \beta$, and $a=(i, g, \lambda) \in S_{\alpha}$, define $a_{\beta}=g \psi_{\alpha, \beta}$, where $\psi_{\alpha, \beta}: G_{\alpha} \rightarrow G_{\beta}$ is a structure homomorphism in the semigroup $G$. Also let $p_{\lambda i}=1_{G_{\alpha}}$, the identity of $G_{\alpha}$ for all $\lambda \in \Lambda_{\alpha}, i \in I_{\alpha}, \alpha \in Y$.

Condition (i) holds trivially. Condition (ii) follows from (A) and $\psi_{\alpha, \alpha}=\iota_{G}$, the identity mapping on $G_{\alpha}$. The two multiplications coincide in view of $p_{\lambda i}=1_{G_{\alpha}}$ for all $\lambda \in \Lambda_{\alpha}, i \in I_{\alpha}, \alpha \in Y$, and the multiplication in $G$. Similarly, condition (iii) follows from (B) and the transitivity of the system of homomorphisms $\psi_{\alpha, \beta}$.

Conversely, let $S$ be as in the theorem and assume that $S$ is orthodox. Since $S_{\alpha}$ is orthodox and $P_{\alpha}$ is normalized for each $\alpha \in Y$, it follows easily that all entries of $P_{\alpha}$ must be equal to $1_{G_{\alpha}}$. Hence $S_{\alpha}$ is a rectangular group and can be written as the direct product $S_{\alpha}=I_{\alpha} \times G_{\alpha} \times \Lambda_{\alpha}$.

For any $\alpha, \beta \in Y$ such that $\alpha \geq \beta$, define a function $\psi_{\alpha, \beta}$ by

$$
\psi_{\alpha, \beta}: g \rightarrow(\alpha, g, \alpha)_{\beta} \quad\left(g \in G_{\alpha}\right) .
$$

We show next that the set $\left\{\psi_{\alpha, \beta} \mid \alpha, \beta \in Y, \alpha \geq \beta\right\}$ can be used as a system of structure homomorphisms for $G=\bigcup_{\alpha \in Y} G_{\alpha}$ making it into a semilattice $Y$ of groups $G_{\alpha}$ which can be used for expressing the multiplication in $S$. 
Indeed, let $\alpha \geq \beta$ and $g, h \in G_{\alpha}$. Then with $a=(\alpha, g, \alpha)$ and $b=(\alpha, h, \alpha)$, we get

$$
\left(g \psi_{\alpha, \beta}\right)\left(h \psi_{\alpha, \beta}\right)=a_{\beta} b_{\beta}=(a \circ b)_{\beta}=(\alpha, g h, \alpha)_{\beta}=(g h) \psi_{\alpha, \beta}
$$

and $\psi_{\alpha, \beta}$ is a homomorphism. Since $(\alpha, g, \alpha)_{\alpha}=g$, we obtain $\psi_{\alpha, \alpha}=\iota_{G_{\alpha}}$. For $\alpha>\beta>\gamma$ and $g \in G_{\alpha}$, we obtain

$$
\begin{gathered}
(\alpha, g, \alpha) \circ\left[\left(\beta, 1_{\beta}, \beta\right) \circ\left(\gamma, 1_{\gamma}, \gamma\right)\right]=(\alpha, g, \alpha) \circ\left(, 1_{\gamma},\right)=\left(, g \psi_{\alpha, \gamma},\right), \\
{\left[(\alpha, g, \alpha) \circ\left(\beta, 1_{\beta}, \beta\right)\right] \circ\left(\gamma, 1_{\gamma}, \gamma\right)=\left(\beta, g \psi_{\alpha, \beta}, \beta\right) \circ\left(\gamma, 1_{\gamma}, \gamma\right)=\left(, g \psi_{\alpha, \beta} \psi_{\beta, \gamma},\right)}
\end{gathered}
$$

which implies $\psi_{\alpha, \beta} \psi_{\beta, \gamma}=\psi_{\alpha, \gamma}$. Hence $G$ has the required form.

Since all sandwich matrix entries are trivial, the multiplication here coincides with that in the theorem.

One can similarly derive the structure theorem for bands of groups given in [2] as well as other structure theorems for special completely regular semigroups.

\section{REFERENCES}

1. A. H. Clifford, Semigroups admitting relative inverses, Ann. of Math. (2) 42 (1941), 1037-1049.

2. A. H. Clifford and M. Petrich, Some classes of completely regular semigroups, J. Algebra 46 (1977), 462-480.

3. M. Petrich, Introduction to semigroups, Merrill, Columbus, Ohio, 1973.

4. _ The structure of completely regular semigroups, Trans. Amer. Math. Soc. 189 (1974), 211236.

5. D. Rees, On semi-groups, Proc. Cambridge Philos. Soc. 36 (1940), 387-400.

6. R. J. Warne, On the structure of semigroups which are unions of groups, Trans. Amer. Math. Soc. 186 (1973), 385-401.

7. M. Yamada, Construction of inversive semigroups, Mem. Fac. Lit. Sci. Shimane Univ. Natur. Sci. 4 (1971), 1-9.

Current address: Department of Mathematics, University of York, Heslington, York YO1 5DD England 